Nachhaltiges Wirtschaften und organisationaler Eigensinn

\title{
Drop Your Tools?
}

\section{Der "Leerformel Nachhaltigkeit" scheint zu gelingen, was ökologische Umbau- programme und Wendeszenarien nicht vermocht haben: Unternehmen, Industrie- und Wirtschaftsverbände präsentieren ein sich wandelndes, mehr strategie-, in- novations- und geschäftsorientiertes Nachhaltigkeitsverständnis. Ihr dabei de- monstrierter Effizienz- und Steverungsoptimismus, der angesichts der hohen Quote gescheiterter Restrukturierungsprojekte anachronistisch wirkt, wird ab- sehbar auf eine "nachhaltige" Probe gestellt werden.}

$\mathrm{D}$ ie Anzeichen, dass nachhaltiges Wirtschaften zum Businessthema wird, verdichten sich: In einer aktuellen Ifo-Umfrage weisen ca. 100.000 Unternehmen in Deutschland eine aktive Nachhaltigkeitspolitik aus. Diese wird zwar überwiegend noch auf die Verlängerung bisheriger ökologischer Unternehmenspolitik reduziert, signalisiert aber eine bemerkenswerte Bereitschaft , ,in Nachhaltigkeit zu investieren“ (1). Diesen auch in internationalen Studien (2) ausgewiesenen Trend erkunden führende Beratungsgesellschaften in einer sicher noch nicht abgeschlossenen Reihe von Buchpublikationen. Nicht ohne Seitenblick auf möglicherweise umsatzträchtige Beratungsthemen wird Unternehmensnachhaltigkeit als zukünftig konkurrenzentscheidende Managementkompetenz propagiert. Das Ziel ist, sich mit langfristig angelegten, ökologisch, ökonomisch und sozial ausbalancierten Geschäftsmodellen gegenüber Kunden und Stakeholdern - darunter nicht zuletzt den Shareholdern - strategisch zu repositionieren (3).

In einem mehrstufigen Berater-Delphi zu den zukünftigen Chancen und Risiken nachhaltigen Wirtschaftens befragt, weisen Umwelt- und Unternehmensberater zwar darauf hin, dass operative Konzepte für ein integriertes Nachhaltigkeitsmanagement erst mittelfristig zur Verfügung stehen. Sie prognostizieren aber seiner dreidimensionalen Integrationsanforderung ,Innovationstreiber-Qualitäten " und Synergiepotenziale für eine zukunftsfähige Geschäftsplanung und Produktpolitik, bei der Integration funktional differenzierter Managementsysteme und beim Redesign interner wie externer Wertschöpfungsprozesse (4).

Dementsprechend breit ist das Instrumentenspektrum, das in einer wachsenden Zahl von Förderinitiativen und Innovationsnetzwerken inzwischen entwickelt und auch von kleinen und mittelstän- dischen Unternehmen getestet wird (5). Bislang ist jedoch weder theoretisch noch praktisch hinreichend geklärt, wie diese praktizierten und prognostizierten Ansätze nachhaltigen Wirtschaftens mit realistischen Erfolgsaussichten zur dauerhaften Unternehmenspraxis werden können.

\section{- Grenzen standardisierter Instrumente}

Nachhaltigkeit in den Mittelpunkt von Unternehmensreorganisationen zu stellen und mit der technisch-ökonomischen Modernisierungsdynamik zu synchronisieren, erfordert bislang kaum erprobte Innovationen des normativen, strategischen und operativen Managements: Unternehmensziele sind neu zu definieren und mit darauf abgestimmten Managementinstrumenten zu operationalisieren; die klassischen Führungs- und Geschäftsprozesse sind neu zu konfigurieren, ihre Ergebnisse sind kontinuierlich zu evaluieren und rückzukoppeln für eine Überprüfung und Verbesserung des strategischen und operativen Managements. Dabei geht es auch um die Anschlussfähigkeit an bisher erfolgreiche Managementpraktiken und Geschäftsmodelle sowie die Moderation der bei fachrationalen Entscheidungsprozessen unvermeidlichen Abstimmungs- und Aushandlungskonflikte. Ein in diesem Sinne erfolgreicher rekursiver Managementzyklus wird mit standardisierten Managementtools und funktional separierten Managementsystemen alleine nicht gelingen.

Modellentwicklung, Erprobung und vergleichende Bewertung nachhaltigkeitsorientierter Instrumente und Indikatoren sind notwendig und unentbehrlich. Entscheidend wird jedoch sein, sie unternehmensindividuell zu konfigurieren, mit bereits vorhandenen (insbesondere den IT- und Controlling-) Systemen zu verknüpfen und perspektivisch in ein nachhaltigkeitsorientiertes, generisches Management der Managementsysteme“ $\mathrm{zu}$ integrieren. Unternehmensindividuell dafür den geeigneten Instrumenten-Mix mit dem dazu gehörenden kontextsensiblen Projektmanagement entwickeln und realisieren zu können, macht die eigentliche Kernkompetenz eines Nachhaltigkeitsmanagements aus. Dass sie als one-best-way weiterzuentwickeln oder als best-practice zu erlernen ist, erscheint eher zweifelhaft: zu spezifisch, kontingent und komplex ist das Bündel an Zielkonflikten, Synergien und Interdependenzen, das mit der strategischen und operativen Balance von ökonomischer Effizienz, ökologischer Innovation und sozialer, gesellschaftspolitischer Verantwortung verbunden ist.

Der als Lernprovokation gedachte Imperativ ,drop your tools" (6) ist also auch beim Nachhaltigkeitsmanagement als Aufforderung zu verstehen, sich auf einen evolutionär-prozessorientierten Lernprozess einzulassen und nicht auf noch so ausgeklïgelte Masterpläne für nachhaltige Unternehmensreorganisationen zu warten. Intensiver als bisher werden Praktiker, Wissenschaftler aber auch Stakeholder also von den technisch-ökonomisch wie mikropolitisch komplexen Prozessen (inter-)organisationaler Koordination, Aushandlung und Entscheidung Kenntnis zu nehmen haben. Mehr als bislang werden sie zu investieren haben in organisationale Regeln und Ressourcen für die mit Nachhaltigkeit verbundenen interessengeleiteten, Umwege einschließenden und rückschlagsbedrohten Such- und Lernprozesse, die über das Schicksal von externen und selbstverpflichteten Nachhaltigkeitsansprüchen entscheiden.

\section{Die Kunst der Rekursivitätshand- habung}

Wie reformgeplagte Unternehmenspraktiker wissen, ist jede Innovation janusköpfig: Neuentwicklungen sind verquickt mit nichtintendierten Folgen und Nebenwirkungen, mit nichtvorhersehbaren Handlungsoptionen und Interessenkonstellationen, die auf Verlauf und Ergebnis der Innovation zurückwirken, einen Großteil der verfügbaren Ressourcen absorbieren und nicht selten ihren Erfolg verunmöglichen. Innovationen und Organisationsreformen sind deshalb kaum linear-sequenziell zu realisieren und gezielt zu optimieren. Erfolgreich sind sie, wenn es gelingt, diese nichtvorhersehbare, geschweige denn kalkulierbare Reflexivität jeder Organisationsreform in rekursiven, wiederholten Schleifen und mit der dafür nötigen Kombination von Expertensicherheit und Prozesssicherheit aufzufangen. Die dabei zu erlernende „Kunst der Rekursivitätshandhabung“ (7) besteht darin, Mittel 
und Wege der Rückkopplung, Kontrolle und Revision, aber auch der Moderation, Abstimmung und des Ausgleichs zu finden und so zur Geltung zu bringen, dass Nichtgewolltes, Nichtbeabsichtigtes, Nichterkanntes und Mehrdeutiges als Innovationsblockade neutralisiert und bestenfalls sogar als Innovationsressource genutzt werden können.

Nachhaltiges Wirtschaften ist mit einem höherem Reflexivitätspotenzial an Unsicherheit, Nichtberechenbarkeit, Komplexität, Konflikthaltigkeit und Mehrdeutigkeit verbunden als andere Unternehmensinnovationen. Daher wird es in besonderer Weise auf diese Kompetenz eines reflexiv sozialen Prozessmanagements angewiesen sein, die keineswegs gleichzusetzen ist mit dem vielzitierten Management ,weicher Faktoren“. Im Vordergrund steht nicht der ominöse menschliche Faktor, sondern der Faktor Organisation, der den Erfolg nachhaltigkeitsorientierten Managements an die im folgenden Schaubild zusammengefassten institutionellen Prämissen knüpft.

Integration und Balance von ökonomischen, ökologischen und sozialen Unternehmensanforderungen sind angewiesen auf eine dauerhafte Synchronisation von Innovations- und Wissensmanagement, Organisations- und Personalentwick-

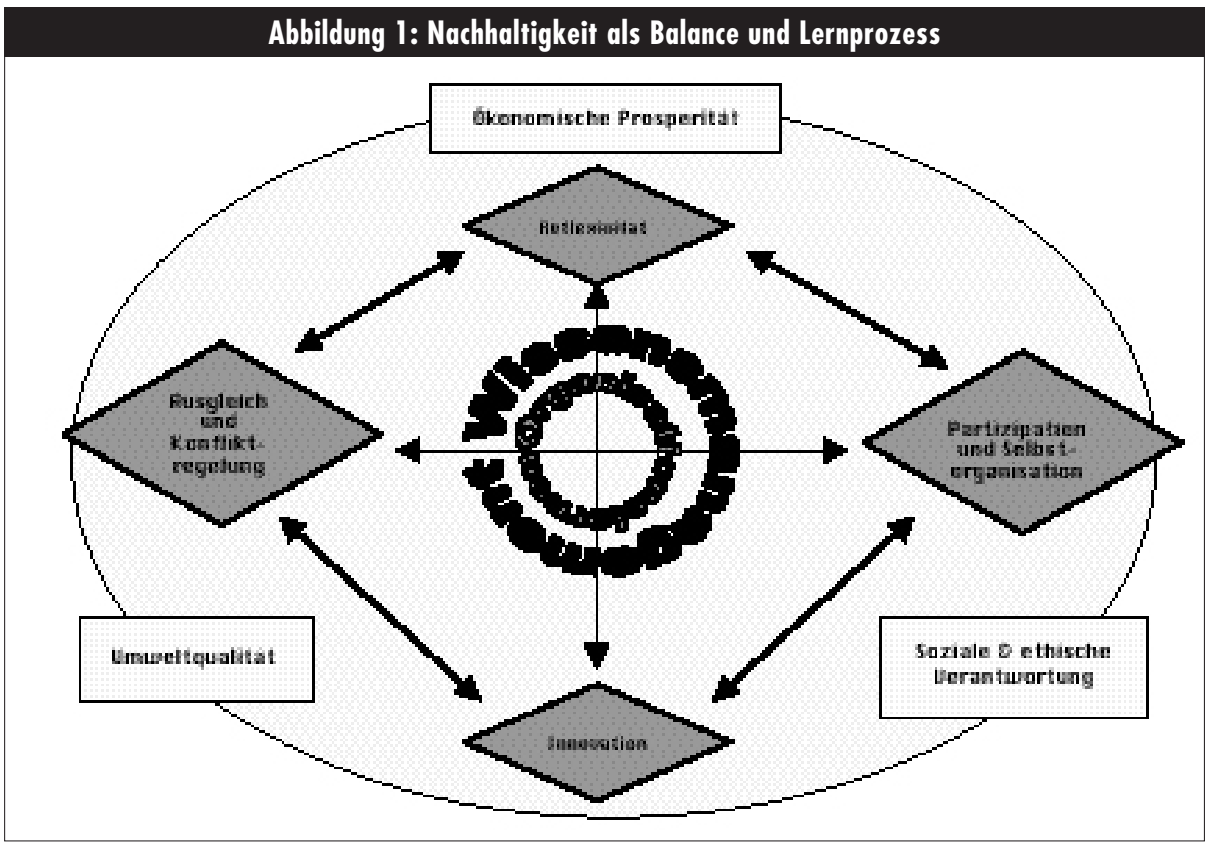

lung: Die kontinuierliche Revision des vorhandenen Wissens und die unentbehrliche Generierung, Verteilung und Anwendung neuen Wissens - zur Zeit vielfach begrenzt auf technisch verbesserte Informationsspeicherung in komplexen ITSystemen und EDV-Architekturen - brauchen ein Human-Resource-Management, das ,Empower- ment" der Mitarbeiter nicht nur proklamiert, sondern Entscheidungsbeteiligung, Partizipationsbefähigung und Mitverantwortung mit Hilfe von fachübergreifenden Qualifizierungsprogrammen, materiellen wie immateriellen Anreizsystemen gewährleistet.

Das dabei unvermeidliche Experimentieren ist ergebnisoffen, und seine interessengeleiteten Abstimmungs- und Aushandlungskonflikte werden keineswegs nur sachrational entschieden. Daher sind Verfahren fachübergreifender Moderation und partikularitätsausgleichender Mediation zu institutionalisieren, die kontext- und problembezogen strategische Handlungsspielräume erschließen und nutzen, keineswegs jedoch auf Kompensation von Besitzständen und Erbhöfen zu beschränken sind.

Organisationale Regeln und Ressourcen zu entwickeln, mit denen diese fachlich wie mikropolitisch unübersichtlichen Lernprozesse unabhängig, wenn auch nicht losgelöst von Personen, Situationen und Handlungskonstellationen erfolgreich zu etablieren sind, zeichnet das jeweils nur unternehmensindividuell mögliche Organisationslernen aus. Es kann deshalb auch als Schlüsselkompetenz für Nachhaltigkeitsmanagement ange-

Quelle: eigene Darstellung

Nachhaltigkeit als Balance und Lernprozess zu verstehen und zu praktizieren, bedeutet also auch, den Rationalitätsmythos des plan- und steuerbaren Organisationswandels nicht wieder zu beleben und sich von einem Nachhaltigkeitsbegriff zu verabschieden, der Zukunftsfähigkeit mit Sicherheit vor künftigen ökologischen, ökonomischen und sozialen Fehlentwicklungen verwechselt.

\section{Anmerkungen}

(1) ifo Institut für Wirtschaftsforschung (Hrsg.): Auswertung der Unternehmensbefragung für das Verbundprojekt "Ökoradar". Endbericht, München 2002.

(2) UNEP: Tomorrow`s Markets - Global Trends and Their Implications for Business, 2002, www.uneptie.org

(3) Figge, Frank/ PricewaterhouseCoopers: Wertschaffendes Umweltmanagement - Keine Nachhaltigkeit ohne ökonomischen Erfolg. Kein ökonomischer Erfolg ohne Nachhaltigkeit, Lüneburg, Frankfurt/Main 2002; Hardtke, Arnd/ Prehn, Marco (Hrsg.): Perspektiven der Nachhaltigkeit, Wiesbaden 2002; Gérard, Jean-Luc: Börse oder Leben, Bern 2002.

(4) Birke, Martin/Göbel, Markus/ Schwarz, Michael: Beratung der Beratung - Umweltmanagement und Umweltberatung im Wandel, Projektabschlussbericht für die HansBöckler-Stiftung, Köln 2001.

(5) Vgl. die in www.zebis.info dokumentierten Beispiele des DBU-geförderten Forschungsprojektes „Nachhaltiges Wirtschaften in kleinen und mittelständischen Unternehmen" und die unter www.ina-netzwerk.de dokumentierten Projekte der Förderinitiative „Betriebliche Instrumente für nachhaltiges Wirtschaften" (INA) des BMBF.

(6) Schein, Karl E.: Drop Your Tools: An Allegory for Organization Studies. In: Administrative Science Quarterly, 41 (1996), No. 6, S. 301-313.

(7) Wimmer, Rudolf: Wider den Veränderungsoptimismus Zu den Möglichkeiten und Grenzen einer radikalen Transformation von Organisationen. In: Soziale Systeme 5 (1999), Nr. 1, S. 159-180; Ortmann, Günther: Das Kleist-Theorem - Über Ökologie, Organisation und Rekursivität. In: Birke, Martin/ Burschel, Carlo/ Schwarz, Michael (Hrsg.): Handbuch Umweltschutz und Organisation, München/Wien 1997, S. 23-91.

\section{Der Autor}

sehen werden. Anders als im konventionellen Effizienzverständnis angenommen schützt auch diese jedoch nicht vor den Unsicherheitszonen nachhaltigen Wirtschaftens, bereitet aber darauf vor und schafft Möglichkeiten, diese nichtroutiniert zu gestalten.
Dr. Martin Birke ist wissenschaftlicher Mitarbeiter im Forschungsschwerpunkt Organisation und Ökologie des ISO-Instituts Köln.

Kontakt: ISO Institut zur Erforschung sozialer Chancen, Neusser Str. 219, 50733 Köln. Tel. 0221/ 8881120-7, E-Mail: martin.birke@iso-koeln.de 
(c) 20I0 Authors; licensee IÖW and oekom verlag. This is an article distributed under the terms of the Creative Commons Attribution Non-Commercial No Derivates License (http://creativecommons.org/licenses/by-nc-nd/3.o/), which permits unrestricted use, distribution, and reproduction in any medium, provided the original work is properly cited. 